\title{
3D imaging of semiconductor colloid nanocrystals: on the way to nanodiagnostics of track membranes
}

\author{
S.I. Kulyk ${ }^{1,2, *}$, I.Y. Eremchev ${ }^{1}$, A.A. Gorshelev ${ }^{1}$, A.V. Naumov ${ }^{1}$, D.L. Zagorsky ${ }^{3}$, \\ S.P. Kotova ${ }^{4}$, V.G. Volostnikov ${ }^{4}$, and E.N. Vorontsov ${ }^{4}$ \\ ${ }^{1}$ Institute for Spectroscopy RAS, 108840 Troitsk, Moscow, Russia \\ ${ }^{2}$ Moscow Institute of Physics and Technology, 141700 Dolgoprudny, Russia \\ ${ }^{3}$ Schubnikov Institute of crystallography, 119333 Moscow, Russia \\ ${ }^{4}$ Samara branch of the Physical institute of RAS, 443011 Samara, Russia
}

\begin{abstract}
The work concerns the feasibility of 3D optical diagnostic of porous media with subdifraction spatial resolution via epi-luminescence microscopy of single semiconductor colloid nanocrystals (quantum dots, QD) $\mathrm{CdSe} / \mathrm{ZnS}$ used as emitting labels/nanoprobes. The nanoprecise reconstruction of axial coordinate is provided by double helix technique of point spread function transformation (DH-PSF). The results of QD localization in polycarbonate track membrane (TM) is presented.
\end{abstract}

Track etched membranes are widely used in industry (high efficiency water filters) and science (metal nanostructure growing, optical analysis of DNA) [1]. However, the study of physical and chemical properties of such porous media is entangled due to the small sizes of pores (hundreds of nanometers and less). Thus methods of electronic microscopy have several restrictions, and probe microscopy (atomic-force microscopy) allows to only characterize the surface, giving no answer for the question of structure and properties of the pores' walls.

In recent years the characterization of materials with complex structure and objects at nanometer scale by methods of super resolution optical microscopy with detection of single luminescence emitter-probes has become more and more popular ([2,3] and links inside). The potential of such methods can be fulfilled in a nano diagnostics of complex polymer structures, and track membranes also. Moreover, the photophysical (spectral) characteristics of such probes are very sensitive to local environment, that can be used in future for characterization of that environment [2-7].

Determination of all three spatial coordinates with nanometer precision is possible due to use of an idea of instrumental transformation of point spread function in double helix scheme using adaptive optics' phase transformation elements [8].

The purpose of introduced work is development of methods of 3D laser spectromicroscopy of single colloid nanocrystals (quantum dots, QD) for nanodiagnostics of track etched membranes.

\footnotetext{
*Corresponding author: sergiy.i.kulyk@gmail.com
} 
In present paper the experimental test of original calculated phase transformation elements (masks), that allow the transformation of point spread function in double helix scheme, was conducted [9]. Also it was shown, that the accuracy of reconstruction of coordinates of quantum dots $(\mathrm{CdSe} / \mathrm{ZnS})$, inserted in liquid solution in polycarbonate track membranes, reaches several dozens of nanometers. The separation between individual images of different molecules in each diffracted limited volume of the sample is possible due to inherent QD property of photoluminescence blinking [10-16].

The important part of the work was the choice of "sample-probe" pair. As far as the accuracy of coordinate reconstruction depends on signal-to-noise ratio, the background luminescence of the sample could significantly spoil or even make impossible the detection of single emitters inside the pores. Due to weak cross-section of QD CdSe/ZnS and polycarbonate TM luminescence spectra the precision of 20-40 nanometer for $400-500 \mathrm{~ms}$ exposure was reached.

Also the feasibility of dynamical processes' diagnostics by introduced method was shown - for example, the visualization of filtration process with the help of markers, in real time with nanometer precision.

Authors acknowledge the support of Russian Foundation of Basic Researches (projects 14-02-00822-a and 16-29-11809-ofi_m). Authors are also grateful professor P.U. Apel (Frank Laboratory of Neutron Physics, Joint Institute for Nuclear Research, Dubna) for attested samples of polymer track etched membranes.

\section{References}

1. V.V. Beriozkin et al., Radiation Measurements 34, 593 (2001)

2. A. Naumov, I. Eremchev, A. Gorshelev, Eur. Phys. J. D 68, 348 (2014)

3. A.V. Naumov, Physics Uspekhi 56, 605 (2013)

4. T. Plakhotnik, E. A. Donley, U. P. Wild, Ann. Rev. Phys. Chem. 48, 181 (1997)

5. T. Plakhotnik, D. Walser, A. Renn, Phys. Rev. Lett. 77, 5365 (1996)

6. T. Plakhotnik, J. Lumin. 83-84, 221 (1999)

7. M. Orrit et al., Faraday Discussions 184, 275 (2015)

8. S. Pavani, R. Piestun, Opt. Express 16, 3484 (2008)

9. S.P. Kotova et al., J. Phys.: Conf. Ser. 740, 012013 (2016)

10. M. Nirmal et al., Nature 383, 802 (1996)

11. P. Frantsuzov, M. Kuno, B. Janko, R. A. Marcus, Nature Physics 4, 519 (2008)

12. A.L. Efros, D.J. Nesbitt, Nature Nanotechnology 11, 661, (2016)

13. T. Plakhotnik et al., Phys. Rev. Lett. 105, 167402 (2010)

14. M.J. Fernee et al., J. Phys. Chem. Lett. 3, 1716 (2012)

15. I.S. Osad'ko, I.Yu. Eremchev, A.V. Naumov, J. Phys. Chem. C 119, 22646 (2015)

16. I.Yu. Eremchev, I.S. Osad'ko, A.V. Naumov, J. Phys. Chem. C 120, 22004 (2016) 